\title{
EXPECTATIVAS POLÍTICAS DE JÓVENES ABSTENCIONISTAS EL CASO DEL DISTRITO ELECTORAL FEDERAL X DE VERACRUZ, MÉXICO
}

\section{Alim Getze Mani Eden Vasquez Feria}

\section{Resumen}

En el presente texto se exponen los resultados del análisis lexicométrico realizado a 130 respuestas a la pregunta “¿Por qué motivo votarías?", aplicada mediante un cuestionario a 131 jóvenes abstencionistas electorales del distrito electoral federal diez, ubicado en la ciudad de Xalapa, Veracruz, México. El objetivo fue explorar sus expectativas en torno a los políticos y la política. Aunque no profundiza en las dimensiones subjetivas, los resultados indican la existencia de diferentes tipos de expectativas, mundos lexicales variantes y empleo de palabras particulares por grupos según escolaridad u ocupación. Los datos proceden de una investigación cuantitativa mayor cuyo objetivo fue captar la representación social del voto de dichos jóvenes abstencionistas electorales.

Palabras clave: jóvenes / expectativa / abstencionismo / mundo lexical / lexicometría.

\begin{abstract}
Political expectations of young abstentionists. The case of the $X$ federal electoral district of Veracruz, Mexico

In the present text we present the results of the lexometric analysis performed on 131 answers to the question 'Why would you vote?', Which was part of a questionnaire applied to the same number of young electoral abstentions of the tenth federal electoral district, located in the City of Xalapa, Veracruz, Mexico. The objective was to explore their expectations around politicians and politics. Although it does not deepen the subjective dimensions, the results indicate the existence of different types of expectations, varying lexical worlds and the use of particular words by groups for schooling or occupation. The data is part of a larger quantitative investigation, which aimed to capture the social representation of the vote of electoral abstinence young voters.
\end{abstract}

Keywords: young / expectations / electoral abstention / lexical world / lexicometry.

Alim Getze Mani Eden Vasquez Feria. Licenciado en Sociología por la Universidad Veracruzana, maestro en Ciencias Sociales por el Instituto de Investigaciones HistóricoSociales de la misma universidad. Actualmente trabaja en el Instituto de Investigaciones en Educación de la Universidad Veracruzana (México).

E-mail: alivasquez@uv.mx 


\section{Introducción}

Actualmente, a nivel internacional, los jóvenes son quienes menos votan en los procesos electorales y más desconfían de la política, además de emplear en menor medida los canales tradicionales para vehiculizar las demandas sociales (IDEA Internacional, 2017; Rial, 2015; Maldonado, 2015). En cambio, se sostiene que los jóvenes participan con mayor intensidad en movimientos sociales $^{1}$ que enfocan sus demandas en cuestiones específicas (Maldonado, 2015; Garcés, 2010; Fernández, 2009; Mannarini, et al., 2008; Becerra, 1996).

Considerando lo anterior, indagamos sobre los mundos lexicales y las expectativas políticas que los motivarían a votar expresadas por jóvenes abstencionistas electorales. Partimos del siguiente supuesto básico: el paso de abstencionista a votante estaría acompañado de expectativas; los abstencionistas apostarían con su voto al cumplimiento de estas, como cualquier otro elector. Así, se empleó el análisis lexicométrico para identificar los tipos de expectativas expresados por 131 jóvenes abstencionistas del distrito electoral federal diez de Veracruz, ubicado en el municipio de Xalapa, México, al responder la pregunta: “¿Si decidieras votar, por qué lo harías?”. Se espera que la información hallada permita comprender el abstencionismo juvenil e incentivar adecuaciones en la oferta electoral.

En el primer apartado se presenta un panorama general sobre el abstencionismo a nivel internacional, en México y en el Estado de Veracruz. En el segundo apartado se expone una breve semblanza de cinco modelos explicativos del abstencionismo. En el tercer apartado se presentan algunas investigaciones sobre las expectativas. Dentro del cuarto apartado se exponen la metodología y los métodos lexicométricos usados para el tratamiento

1 Profundizar en la participación política juvenil en México es un gran reto, específicamente en lo que respecta al abordaje de las dimensiones de la movilización social juvenil. De acuerdo con datos del Observatorio de Juventud para América Latina y el Caribe, solo un 30,5\% de los jóvenes encuestados en México indicó participar en alguna organización social. De ese porcentaje, la mayoría cuenta con estudios de secundaria $(4.730 .523,6)$, seguida por quienes indicaron estudios de nivel superior (3.612.768,2). Más allá de esos datos, sabemos poco sobre la participación y activismo juvenil en México, dado que en este tema se enfrenta el enorme reto de superar los problemas relacionados con la medición del fenómeno asociativo en el país. Felipe Hevia (2012) señala que la medición del fenómeno del asociativismo en México es complicada debido al empleo de determinadas denominaciones (qué se entiende por organizaciones de la sociedad civil, por ejemplo) y la manera de contabilizar a partir del reconocimiento legal. 
de la información. En el quinto apartado se presentan los resultados. Para culminar, se desarrolla un apartado que incluye las reflexiones con relación a los hallazgos.

\section{Abstencionismo electoral}

La abstención electoral es un fenómeno presente tanto en democracias consolidadas como en desarrollo. Mizrahi (2015), empleando datos del Instituto Internacional para la Democracia y la Asistencia Electoral (IDEA), identificó los diez países con mayor abstencionismo, ubicados en distintas latitudes ${ }^{2}$. Además, según IDEA, la participación electoral promedio en el mundo se redujo un 10\% durante las tres últimas décadas (Mizrahi, 2015).

Se ha registrado la constancia de la ausencia de los jóvenes en diferentes llamados a las urnas. Ejemplo de ello son el Brexit ${ }^{3}$ y las elecciones presidenciales del 2016 en Estados Unidos. En el primer caso, la abstención fue del 28,7\%, mientras que en el segundo rondó el 45\%. En ambos casos, los resultados generaron muestras de descontento por parte de los jóvenes en sus respectivos países. Paradójicamente, de acuerdo con información periodística, el voto de los jóvenes podría haber cambiado el resultado de uno ${ }^{4}$ u otro caso $^{5}$. Un ejemplo más: Camas (2016) indica que los jóvenes españoles son el colectivo con mayor proclividad a abstenerse electoralmente.

Para el caso mexicano, los hallazgos coinciden: son los jóvenes quienes en menor medida participan en las elecciones (INE, 2016; IMJUVE, 2013; De la Paz y Gómez, 2012). En las elecciones federales del 2012 y las elecciones legislativas del 2015, los ciudadanos con un rango de edad entre los $20 \mathrm{y}$ 29 años fueron los que mayormente se abstuvieron (INE, 2016; IFE, 2013). En el Estado de Veracruz se registró similar resultado en ambas elecciones (IFE, 2013, p. 19; INE, 2016, p. 24).

Además de la característica señalada, los jóvenes comparten otras. Olesker (2016) señala que en Latinoamérica la tasa de desempleo es superior entre los jóvenes. El secretario general adjunto de la Organización Iberoamericana de la Juventud (OIJ) indica que los jóvenes iberoamericanos enfrentan un panorama complejo, compuesto por retos e incertidumbres en temas como educación, familia, empleo, violencia y salud, con características diferentes

2 Los países en cuestión son Chile, República de Eslovenia, Mali, Serbia, Portugal, Lesoto, Lituania, Colombia, Bulgaria y Suiza (Mizrahi, 2015).

3 Brexit es una abreviatura de dos palabras en inglés, Britain (Gran Bretaña) y exit (salida), que refiere a la salida del Reino Unido de la Unión Europea, en el marco del referéndum correspondiente a esta decisión.

4 Para el caso de las elecciones presidenciales 2016 en Estados Unidos, véase la nota periodística de López (2016).

$5 \quad$ Para el caso del Brexit, véase Sota (2016). 
a las experimentadas al menos por dos generaciones anteriores (OIJ, 2013). El Instituto Mexicano de la Juventud (IMJUVE) indica que el principal problema al que se enfrenta la juventud mexicana es su incorporación a la vida social y productiva del país en condiciones desfavorables (IMJUVE, 2013).

Para Saraví (2015), los jóvenes se incorporan al trabajo, la escuela, los procesos electorales, entre otros, en y desde condiciones sociales que limitan su desarrollo individual y colectivo, situación a la cual denomina inclusión desigual. El abstencionismo electoral juvenil podría entenderse como el resultado de las condiciones en las que viven los jóvenes, sin embargo, los estudios en torno al abstencionismo han desarrollado modelos explicativos diversos.

\section{Perspectivas teóricas}

El abstencionismo electoral es considerado tanto una acción en el sentido negativo, del no hacer (Bobbio, 2001), como la expresión de una determinada voluntad (Oñate, 2010). Debido a su comportamiento complejo y cambiante (Giraldo, 2012), ha llamado la atención de los investigadores e instituciones (Jiménez, 2015). En las investigaciones en torno al abstencionismo se encuentran ecos de cinco modelos explicativos. El primer modelo explicativo se desprende de los aportes de la Escuela de Columbia, los cuales subrayan los elementos de integración que fungen como variables explicativas de la participación electoral (Ochoa, 2015). Considerando las variables socioeconómicas y demográficas, la apuesta es encontrar regularidades en el empleo, la escolaridad, el ingreso, el lugar de residencia, para delinear el perfil de los abstencionistas (Morales, et al., 2011) y encontrar los determinantes socioeconómicos de la abstención. Por ejemplo, Chavarría (2014) reportó una relación positiva entre el índice de desarrollo humano y la participación electoral en Costa Rica en el 2010; en una publicación de la Organización de los Estados Americanos (OEA, 2014) que aborda el caso de América Central se señala: la participación política y electoral se asocia con el nivel de desarrollo humano (OEA, 2014).

El segundo modelo explicativo se desarrolla a partir de la Escuela de Michigan. Busca develar las características socioculturales generales de los abstencionistas, para lo cual se analizan las actitudes políticas aprendidas en el desarrollo de su vida y el proceso de socialización, además de los valores y creencias del individuo. Destaca la identidad partidista como variable explicativa del voto o la abstención (Ochoa, 2015). Este modelo, denominado psicológico, se conforma con variables blandas - sin dejar de lado las variables duras - y el llamado embudo de causalidad es uno de sus aportes más conocidos (Sulmont, 2010).

Un tercer modelo explicativo se desarrolla a partir de la teorización de la elección racional. Al decir de Barry (1970), la obra de Downs (1957) sienta las bases del argumento del costo-beneficio para la elección entre acudir o 
no a las urnas. Una competitividad escasa, o la percepción de ella por los electores, desalentará la participación electoral al desvalorizar el voto (Barry, 1970). Fiorina (1981) sostiene la relación entre la percepción de la situación socioeconómica y la participación electoral en la noción del voto retrospectivo para explicar la abstención como resultado de cálculos de costo-beneficio realizados por los electores. Boix y Riba (2000), al analizar el abstencionismo en España, subrayan la importancia explicativa del grado de competitividad electoral.

El cuarto modelo explicativo es el institucional. Características como el sistema de partidos, el sistema electoral y la administración electoral cobran relevancia para comprender la inasistencia a las urnas. La explicación recae en las características y procesos institucionales externos al individuo que configuran las condiciones bajo las cuales los sujetos optan o no por participar electoralmente (Nohlen, 1994). North (1989) se sitúa en este espectro al sostener la configuración de las reglas del juego por parte de las instituciones y subraya el sentido del deber como catalizador de la acción de participación electoral por encima del cálculo racional. Nohlen busca complementar este modelo con el análisis del contexto como un producto histórico para logar comprender la decisión del votar o abstenerse (Nohlen, 2003).

Un quinto modelo sostiene que la participación electoral se relaciona con los factores del ciclo de vida. La participación electoral aumenta con el cumplimiento de años del elector en conjunción con la adopción del rol de adulto (Leppäniemi, et al., 2010; Wattenberg, 2003). El votar no forma parte de las perspectivas y necesidades propias del ciclo de vida que atraviesan los jóvenes (Seagull, 1971). Los trabajos de Lazarsfeld, Berelson y Gaudet (1962) y de Anduiza y Bosch (2004) son muestra de la influencia de este modelo.

Son mencionables otros trabajos que, por sus características, difícilmente podrían ser encuadrados en algún modelo previamente descrito. Almond y Verba (1963), por ejemplo, sostienen que las orientaciones de los ciudadanos respecto a su sistema político incluyen elementos cognitivos, evaluativos y afectivos; la forma en que estos se combinan explica el comportamiento político incluyendo el votar o no. Además, dichos elementos son la base para sostener la existencia de tres tipos de cultura política (parroquial, de súbdito y participativa), las cuales pueden ser mixtas, es decir, contener elementos de las otras — salvo el caso de la cultura parroquial- . Las culturas políticas son analizadas en su congruencia con respecto a las estructuras de los sistemas políticos: existe una situación de congruencia caracterizada como lealtad cuando se equilibran las actitudes y las instituciones; en cambio, estamos ante una situación de incongruencia denominada alineación cuando las actitudes tienden a rechazar las instituciones (Almond y Verba, 1963). La no congruencia entre la cultura política y el sistema político explicaría el abs- 
tencionismo electoral, por tanto, se torna necesario identificar los elementos que conforman a una y a otro.

Burden (2009) reflexiona sobre la relación entre escolaridad y participación electoral, en el marco del problema del rompecabezas de Brody ${ }^{6}$. De acuerdo con el modelaje que emplea, los cambios en la relación se explican aceptando el dinamismo de los efectos de la educación sobre la participación (Burden, 2009). El signo de la relación entre las variables mencionadas cambia, por lo cual es menester reflexionar sobre los resultados bajo el contexto en el que se presenta uno u otro signo de relación. Esto invita, siguiendo al autor, a explorar la capacidad explicativa de la teoría de la educación cívica, que sostiene la influencia de la educación formal en incrementar o decrementar la probabilidad de abstenerse dada la instrucción en las aulas, a la par del acceso - como universitarios - a redes sociales articuladas en las cuales se socializa el sentido del deber cívico (Burden, 2009).

Dada la afinidad de los cinco modelos con cuatro disciplinas (sociología, psicología social, estadística social y ciencias políticas), se ha conformado un corpus teórico-metodológico complejo. Además, se ha acumulado una serie de resultados que refuerzan uno u otro modelo. Para el caso mexicano, la escolaridad se ha identificado como variable relacionada tanto positiva como negativamente con la abstención (Morales, et al., 2011, p. 36). Murillo (2009) muestra "[...] una relación estadística significativa (para las elecciones de 2000 y de 2001 en Ciudad Juárez, Chihuahua) entre la abstención, la edad, los ingresos, la escolaridad y la migración" (Morales, et al., 2011, p. 27).

Los estudios que emplean información proveniente de abstencionistas son pocos. En una revisión breve de la producción contemporánea en México tan solo se encontraron dos tesis de licenciatura (Martínez, 2013; De la Rosa, 2010), una de doctorado (Jiménez, 2009), una ponencia (Murillo y Pulido, 2009), un artículo (Fernández, et al., 2011), además de un libro (Padilla, 2010). Quizá esto se deba a la gran dificultad para identificar a los abstencionistas y la escasa información institucional respecto a ellos.

Los estudios sobre el abstencionismo ofrecen elementos importantes para considerarlo un fenómeno multifactorial que no responde a leyes universales de causalidad (Giraldo, 2012). En este orden de ideas, se busca profundizar en una arista explicativa del abstencionismo juvenil como una expresión del desencuentro entre las instituciones tradicionales de la política (partidos políticos) y las expectativas político-electorales de los jóvenes (Makowski, 2008). Considerando esto, se realizó una revisión de algunos

6 Burden lo describe de la siguiente manera: "los académicos ya sabían que (1) la educación predice fuertemente la participación electoral y que (2) los niveles agregados de educación aumentaron drásticamente, pero no se siguió que (3) la participación electoral aumentó" (Burden, 2009, p. 542). 
trabajos que han estudiado las expectativas con la finalidad de identificar los elementos teóricos básicos para poder explorar las expectativas políticas de los abstencionistas juveniles.

\section{Expectativas}

Desde hace poco más de una década, los investigadores sociales han mostrado interés por las expectativas como factores explicativos del comportamiento económico, político o escolar (OIJ, 2013). En el ámbito de la investigación cuantitativa, las expectativas ya eran consideradas un factor explicativo, pero no se medían de manera directa (OIJ, 2013). Su medición llega de la mano de la valoración de las variables "blandas"7 en la construcción de indicadores que complementen la información derivada de las variables sociodemográficas, como los indicadores subjetivos de bienestar y felicidad promovidos por el Banco Mundial (Graham, 2010), además del índice de expectativas juveniles (OIJ, 2013).

Otro de los objetivos de la investigación cuantitativa de las expectativas es diseñar tipologías. Por ejemplo, Sander, Stevenson, King y Coates (2000) reportan tres tipos de expectativas encontradas en alumnos de educación superior: expectativas predictivas (lo que es más probable que ocurra), expectativas normativas (lo que se espera que ocurra con base en la experiencia de situaciones similares) y expectativas ideales (lo deseable) (Sander, et al., 2000).

Los estudios cualitativos se han enfocado en indagar el proceso de conformación de las expectativas. Archer (2007) estudia el proceso reflexivo individual por el cual se eligen cursos de acción futuros. Los individuos reflexionan sobre las condiciones de su entorno inmediato, reconociendo conjuntamente límites y restricciones, además de facilidades y oportunidades para la realización de sus proyectos. Ello sucede gracias a una "conversación interna" en la cual los sujetos definen, redefinen y estructuran aspiraciones y metas, conforman planes y establecen un modus vivendi compuesto por un conjunto de prácticas satisfactorias (Archer, 2007). Las expectativas se adecuarán en relación con el contexto en el que se efectúan los actos para su consecución.

La propuesta teórica y resultados de investigación de Chalari permiten conocer más sobre los arreglos entre las expectativas individuales y las sociales. La autora analiza la mediación, a la que comprende como el modelo específico que explica las posibles formas en las que las expectativas individuales se conectan con las sociales (Chalari, 2012 y 2009). El tipo de relación entre ambas dependerá de su conjunción bajo un contexto, dando lugar a tres formas. La primera de ellas surge cuando las preocupaciones personales se

7 Variables relacionadas con la percepción subjetiva otorgada a determinados objetos, situaciones o procesos. 
enfrentan con las expectativas sociales, en un contexto social predominantemente restrictivo. La segunda emerge de la conjunción de expectativas sociales complementarias de las personales, en un contexto favorable. La tercera forma sucede cuando, en contextos neutrales, las expectativas sociales no se configuran como preocupaciones personales (Chalari, 2009). En el proceso de resolución de las tensiones individuo-grupo, las expectativas individuales pueden verse modificadas, acercándose o alejándose de las expectativas sociales, produciendo un paso de estrategias de acción conciliadoras a combativas. En síntesis, es posible realizar tipificaciones de las expectativas, ya sea en un estudio cuantitativo o cualitativo. La tensión a la que aluden tanto Archer como Chalari entre lo individual y lo social dentro de un contexto es un elemento teórico útil para definir el tipo de expectativas.

\section{Metodología}

El presente trabajo reporta parte de los hallazgos de una investigación realizada por el autor en 2017, intitulada La representación social del voto en jóvenes abstencionistas del distrito electoral federal diez de Xalapa, Veracruz. Dicho estudio fue de tipo cuantitativo, exploratorio y transversal; se empleó la técnica de encuesta y un cuestionario compuesto por 24 preguntas, de las cuales 4 eran abiertas. Los sujetos de investigación fueron jóvenes abstencionistas, entendiendo por estos a aquellas personas con un rango de edad de entre 18 y 29 años, inscritas en lista nominal y que no ejercieron su derecho al voto en una o más elecciones - ya fueran de tipo local, federal o extraordinarias - realizadas en dicho distrito electoral.

Para el cálculo de la muestra se empleó la siguiente información. De acuerdo con el Instituto Nacional Electoral (INE) un total de 82.373 personas con una edad entre 18 y 29 años estaban registradas en la lista nominal del distrito aludido. A nivel estatal, en 2009, el 45\% de los jóvenes sí votó, mientras que el 55\% no votó, de acuerdo con información del Centro de Estudios Sociales y de Opinión Pública (CESOP, 2012, p. 9).

Esta información se usó en la siguiente fórmula para el cálculo de la muestra:

$$
\mathrm{N}=\frac{N p q}{(N-1) D+p q} \quad \mathrm{y} \mathrm{D}=\frac{\mathrm{B}^{2}}{z^{2}}
$$

$\alpha / 2$

Se sustituyen los valores considerando $\mathrm{B}=0,085$ el error permitido en la estimación de la proporción de encuestados y z_ $(\propto / 2)^{\wedge} 2=3,8416$ el valor de tablas usando la confiabilidad de $\alpha=0,05$, o sea, con un $95 \%$ de confiabilidad. 
El resultado fue 131 jóvenes a quienes aplicar el cuestionario. Se determinó aplicarlo en las 20 secciones electorales con menor participación ${ }^{8}$, las cuales estaban ubicadas en tres zonas: noreste, noroeste y suroeste de Xalapa. Las dos primeras zonas están compuestas predominantemente por Áreas Geoestadísticas Básicas (AGEB) de alta marginación; en tanto que la zona sureste la componen AGEB de media y baja marginación, de acuerdo con los datos del Consejo Nacional de Población (CONAPO, 2010).

$\mathrm{Al}$ carecer de información institucional o académica sobre el domicilio de los jóvenes abstencionistas, la aplicación del cuestionario se realizó de la siguiente manera. Una vez en las calles que conforman las secciones electorales, se llamaba en la entrada de cada casa y se consultaba por alguna persona que reuniera los criterios de inclusión. De ser positiva la respuesta y si el joven abstencionista se encontraba presente, se le aplicaba el cuestionario. En caso de que no estuviera presente, se agendaba una cita para su posterior aplicación.

Debido a las dificultades derivadas de ubicar a los abstencionistas juveniles en campo, solo se cubrió el $91 \%$ de la muestra, en tanto que el 9\% restante se completó con un levantamiento en lugares públicos que mantuvo los criterios de inclusión y añadió el requisito de que los encuestados pertenecieran a una sección electoral ubicada en el distrito electoral federal diez. Se optó por realizar lo anterior dado que permitía cumplir con el diseño muestral planteado alterando mínimamente la cuota por secciones.

Para construir tipologías con información procedente de preguntas abiertas se empleó la lexicometría, rama de la estadística que permite el análisis cuantitativo de los textos, en conjunto con los aportes de Reinert (1986), quien propuso la noción de "mundo lexical" a partir del análisis estadístico de la organización y distribución de la sucesión de palabras que componen los enunciados de un texto (el cual puede ser la transcripción de una entrevista o de la respuesta abierta en un cuestionario estructurado) tomando en cuenta la "coocurrencia" de varias palabras principales en un mismo enunciado, "técnicamente los mundos lexicales son un conjunto de palabras principales que tienen una organización habitual (repetitiva) en el discurso y que se refieren a algo similar" (De Alba, 2004, p. 14).

Una de las técnicas que son de gran ayuda para lograr captar los mundos lexicales es el árbol de similitud: "[...] técnica que permite identificar coocurrencias entre las palabras según sus conexiones en el texto, ayudando a identificar la estructura del contenido del corpus textual gracias a su visualización en forma de gráfico" (Marchand y Ratinaud citados en Molina, et al., 2017, p. 94). A mayor frecuencia de una palabra, mayor es su tamaño

8 Estrategia que ha mostrado viabilidad en los trabajos de Padilla (2010), Jiménez (2009) y Murillo y Pulido (2009). 
en la representación, mientras que, a mayor coocurrencia en las palabras, más grueso es el enlace entre ellas. Además, el árbol de similitud ofrece la posibilidad de identificar el uso de determinadas palabras según determinadas variables como escolaridad, edad, empleo, etcétera. En conjunto con el árbol de similitud, se emplea el Análisis Factorial de Correspondencias y Especificidades (AFCyE), que permite identificar o reforzar los resultados de asociación entre determinadas palabras y las variables de sus enunciantes (Molina, 2017). Ambos procesamientos requieren la lematixación ${ }^{9}$ previa de la base de respuestas, lo cual, junto con las técnicas descritas, fue realizado para el conjunto de respuestas a la pregunta abierta " ¿Si decidieras votar, por qué lo harías?”, empleando el software Iramuteq en su versión 0.7 Alpha 2.

\section{Resultados}

De los 131 encuestados, el 66\% fueron mujeres; el 59\% tenía entre 19 y 23 años ${ }^{10}$. La mayoría contaba con estudios de nivel superior (54); en segunda instancia, con estudios de nivel básico (39); y en tercera instancia, de nivel medio-superior (37). Las principales ocupaciones reportadas fueron estudio (44) y labores del hogar (40), esta última indicada solo por mujeres ${ }^{11}$. Se diseñó una escala en torno al nivel de conocimiento de los encuestados sobre las reglas e instituciones electorales y los partidos políticos que compitieron previamente $^{12}$. La mayoría de los encuestados (94) cuenta con conocimientos mínimos, mientras que 5 mostraron conocimientos intermedios, 10 registraron un nivel alto y únicamente 2 alcanzaron el nivel experto; los 20 restantes no respondieron acertadamente a alguna de las preguntas.

Las principales palabras empleadas por los 130 jóvenes abstencionistas ${ }^{13}$ para responder a la pregunta “¿Si decidieras votar, por qué lo harías?" fueron: propuesta (20), candidato (14), cambio (13), mejor/mejora (12), político (10), partido (7), realmente (6), hacer (5) y candidatos (5). El conjunto de palabras indica que, a nivel grupal, las expectativas giran en torno a buenas propuestas, un cambio político y candidatos o partidos políticos que cumplan. El reporte de las frecuencias de palabras deja entrever la existencia de una pluralidad en cuanto a las expectativas, al reconocer la convergencia

9 Proceso de reducción de las palabras con base en sus raíces (Molina, 2017).

10 Es preciso recordar la ausencia de información pormenorizada sobre los abstencionistas juveniles, motivo por el cual se desconocía de antemano su composición por rangos de edad.

11 Además de trabajador no formal (16), privado (8), público (8), estudia y trabaja (6), no estudia y no trabaja (4), profesionistas por cuenta propia (3) y empresario (1).

12 Se usaron tres preguntas, cada una con un valor relacionado con su nivel de dificultad. La primera pregunta aportaba 2 puntos, la segunda, 4 y la tercera, 6 . Para identificarlos se empleó la siguiente escala: de 0 a 1 puntos: ínfimo; de 2 a 4 puntos: conocimiento bajo; de 5 a 7 puntos: conocimiento medio; de 8 a 10 puntos: conocimiento alto; de 11 a 12 puntos: conocimiento experto.

De los 131 jóvenes encuestados, uno no respondió la pregunta realizada. 
de elementos específicos como las "propuestas", con aquellas más generales como el "cambio". En la Ilustración 1 se muestra que las expectativas expresadas se agrupan en cinco conjuntos, cada uno de los cuales presenta una organización particular, con relación a la cual se les puede denominar (de derecha a izquierda) las "buenas propuestas", "el cambio político", "la practicidad", el "cambio general" y "cumplimiento del derecho".

\section{llustración 1. Árbol de similitud por cofrecuencia de palabras en las respuestas.}
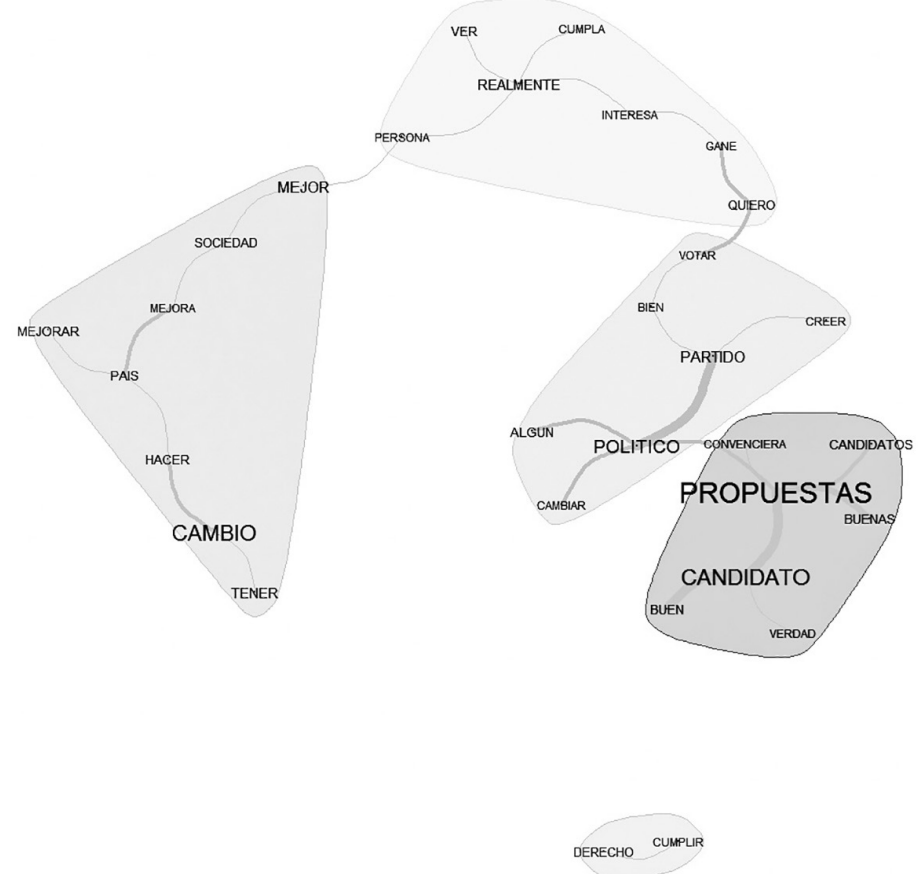

Fuente: Árbol de similitud generado por Iramuteq.

El sentido grupal de cada comunidad se compone por sentidos particulares. A continuación, se detallan las características de cada comunidad, además de las expresiones que le dan identidad y sentido. La comunidad "buenas propuestas" refiere a expectativas con relación a la agenda del candidato y el efecto del convencer para dinamizar el acto de votar. Se relacionan con una actitud evaluativa de la agenda o del conjunto de propuestas.

Algunas de las respuestas en esta comunidad son:

Caso 26: "Que encontrara un candidato con la convicción, compromiso social y que mantuviera como campañas propuestas que reflejen la realidad de las problemáticas de la sociedad, así como sus escenarios para dar solución a estas". 
Caso 127: "Porque estaría convencida de las propuestas y además haría una revisión con sus antecedentes y comprobar que no tenga antecedentes criminales".

La comunidad "cambio político" indica expectativas en los partidos o políticos como agentes de cambio de la situación en la que se perciben los encuestados:

Caso 1: "Para evitar que un partido político obtuviera una gubernatura o la presidencia de la república".

Caso 22: "Para apoyar al partido político que apoye a la gente".

La comunidad "certidumbre" indica expectativas en las que el detonante de la acción del votar es la apuesta segura por el triunfo de la persona que garantizará el cambio. Se compone por aquellas expresiones que subrayan, además del convencimiento, una condicionante del votar:

Caso 42: "Realmente cumpliera [la persona votada] con las promesas que de un discurso convincente".

Caso 123: "Sería una persona realmente confiable que cumpla".

La comunidad "cambio general" implica expectativas más amplias, como lo es un cambio con efectos positivos en el país o en la sociedad. En su interior se encuentran expresiones tendientes a realzar la parte del "deber ser" de los políticos:

Caso 77: "Por que 'hubiera' [sic] un cambio en la política, uno real, que no mientan".

Caso 114: "Un cambio en la sociedad, mejor que ellos se interesen por las necesidades de las personas".

La comunidad "cumplimiento" se conforma con expectativas del ejercicio del derecho a votar. A nivel general, las respuestas sugieren una actitud evaluativa y/o escéptica de los jóvenes abstencionistas. Las respuestas van desde aquellas que subrayan la certidumbre hasta aquellas que apuestan por el cambio general. Para saber acerca de las características de los emisores se procedió a realizar un AFCyE bajo el supuesto de que las convergencias y divergencias de los arreglos dependerían de ciertas características de los emisores, las cuales influirían en la elección de unas palabras ante otras.

Las palabras mayormente empleadas en los dos grupos con mayor presencia en la muestra, estudiantes y encargadas de las labores del hogar fueron "cambio/convenciera" y "gane/cumpla", respectivamente. Mientras que el primer binomio sugiere una aspiración grupal al "cambio", también implica una actitud evaluativa de las propuestas difundidas por los candidatos ("convenciera"). 
Tabla 1. Expresión de palabras por ocupación.

\begin{tabular}{lcccc}
\hline \multicolumn{1}{c}{ Palabra } & \multicolumn{4}{c}{ Grupos } \\
\hline $\mathbf{n = 1 3 0}$ & Estudiante & $\begin{array}{c}\text { Estudia } \mathbf{y} \text { labores } \\
\text { del hogar }\end{array}$ & $\begin{array}{c}\text { Estudia } \mathbf{y} \\
\text { trabaja }\end{array}$ & $\begin{array}{c}\text { Labores del } \\
\text { hogar }\end{array}$ \\
\hline Cambio & $\mathbf{0 , 9 2 6 5}$ & 1,2905 & $-0,1627$ & $-1,2959$ \\
\hline Convenciera & 0,8345 & $-0,0076$ & $-0,0539$ & $-0,7247$ \\
\hline Cumpla & $-0,9808$ & $-0,0076$ & $-0,0539$ & 1,1157 \\
\hline Gane & $-0,9808$ & $-0,0076$ & $-0,0539$ & 1,1157 \\
\hline
\end{tabular}

Fuente: Resultado del AFCyE en Iramuteq.

El binomio "gane/cumpla" apunta a una actitud condicionante del voto por parte de los emisores, que en este caso fueron aquellas mujeres que indicaron por ocupación "labores del hogar". Se realizaron dos árboles de similitud más, con la intención de profundizar los resultados del AFCyE. La Ilustración 2 muestra que quienes indicaron una ocupación de estudiantes se caracterizan por el empleo de la palabra "convenciera", en tanto que quienes indicaron por ocupación labores del hogar hacen lo propio con las palabas "gane" y "cumpla".

Ilustración 2. Frecuencia de empleo de palabras por ocupación.

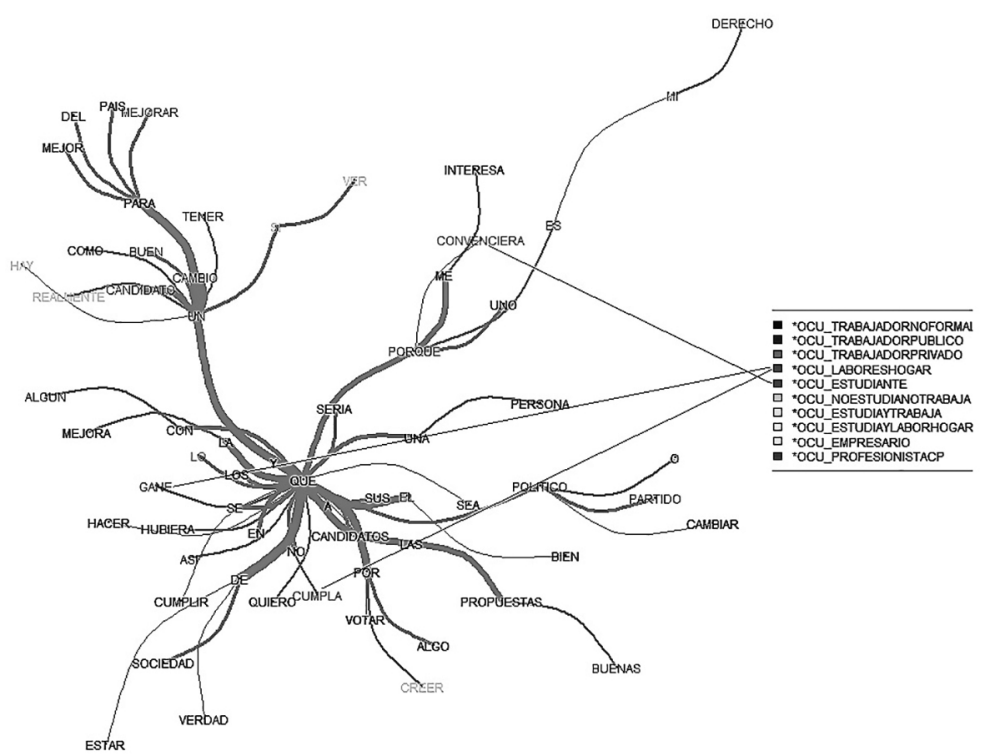

Fuente: Árbol de similitud generado por Iramuteq. 
La Ilustración 3 muestra que quienes cuentan con educación básica emplearon con mayor frecuencia la palabra "persona" y, en cambio, quienes cuentan con estudios de nivel medio superior emplearon "candidato" y los de nivel superior, "derecho". Esto sugiere que quienes cuentan con estudios de nivel básico enfocan sus expectativas en la representación personalista, los del nivel sucesivo lo hacen en una figura más general, como el candidato, $y$, finalmente, los de nivel superior remiten al marco normativo.

Ilustración 3. Frecuencia de empleo de palabras por escolaridad.

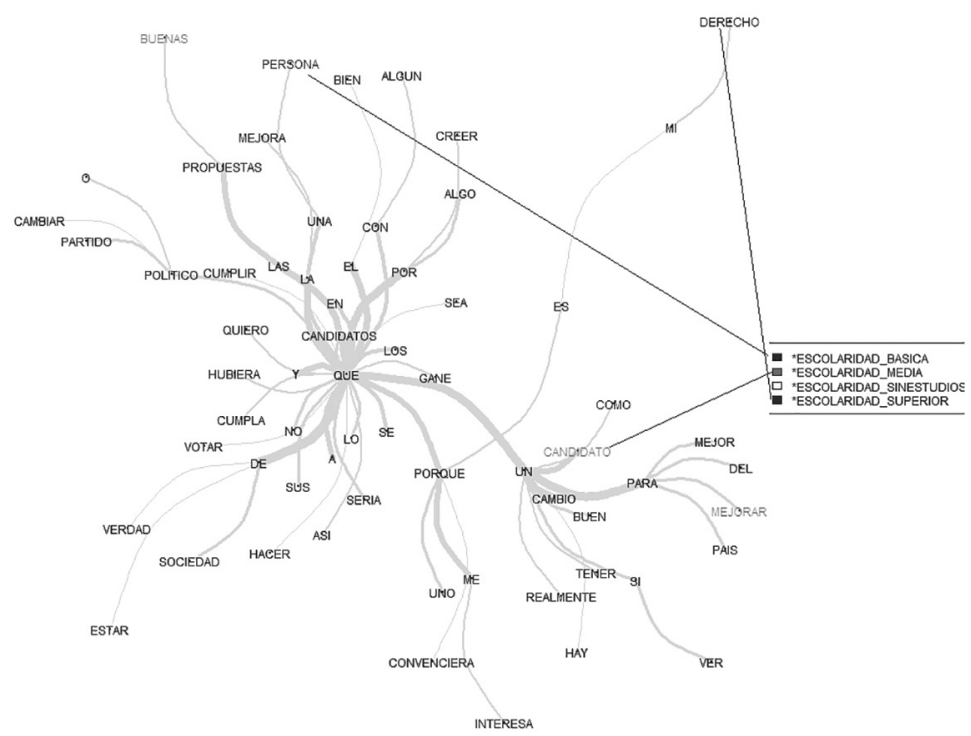

Fuente: Árbol de similitud generado por Iramuteq.

La mayoría de las personas con escolaridad básica son "amas de casa" y la mayoría de los estudiantes cursa o cuenta con estudios de nivel superior, por lo cual podemos considerar la presencia de determinadas palabras como un indicador de la influencia de la escolaridad y ocupación. Siguiendo la reflexión de Burden (2009), es posible sostener que la pertenencia a un nivel escolar y una determinada ocupación indica la inserción de los sujetos en una red social, en la que la convivencia y la interacción propician cierto tipo de expectativas.

\section{Conclusiones}

La exploración de las expectativas en los encuestados arrojó que existen cinco tipos. Si bien en sentido general la mayoría de las expectativas se orienta al "cambio", el análisis del árbol de similitud mostró que existen comunidades con características de sentido y coherencia propias: "buenas propuestas", 
"cambio político", "certidumbre", "cambio general" y "cumplimiento". Las expectativas de los jóvenes abstencionistas guardan relación con la oferta electoral - excepto aquellas que apuntan al "cambio general"- desde un posicionamiento un tanto escéptico con respecto a esta y al compromiso de los actores políticos. En parcial concordancia con Makowski (2008), es posible sostener la existencia de una tensión entre las expectativas de los jóvenes y los partidos políticos, la cual denota una inconformidad que podría ser solventada al modificar la oferta electoral. Esto es consistente con la desconfianza juvenil ante dichas instituciones, captada por el IMJUVE (2013) a nivel nacional e incluso por las reflexiones teórico-explicativas para el abstencionismo juvenil (Giraldo, 2012).

El análisis de las expectativas expresadas permitió identificar características particulares en el empleo de palabras, relacionadas con la escolaridad y la ocupación de quienes respondieron; este hallazgo encuentra consonancia con el modelo sociológico, no obstante, se considera que ambas remiten, más que a un estatus, a las relaciones sociales en las que se encuentran insertos, es decir, apuntan a sus formas de vida para explicar la sofisticación (o particularidades) de las respuestas conforme varían el nivel escolar y la ocupación.

Es de suponer que las interacciones en sus contextos, ya sea la escuela, el trabajo o el hogar, ofrecen los elementos para posicionarse ante temas específicos. No se argumenta que la pertenencia a un nivel escolar o actividad implique un determinado nivel de conocimientos sobre la política y los procesos electorales; en cambio, se sostiene que la adscripción a estos grupos implica asumir ciertas formas actitudinales, las cuales fungen como elementos estructurantes que permiten a los sujetos asumir posicionamientos aun cuando poseen información insuficiente (Parales y Vizcaíno, 2007). Lo anterior hace posible dar cuenta de una situación aparentemente contradictoria: escolaridad de nivel superior junto con un nivel básico de conocimientos sobre el voto y los procesos electorales. Similar resultado encontró Murillo: los ciudadanos cuentan con escasos conocimientos sobre política. Lo que lo llevó a señalar la existencia de un analfabetismo político en el caso de Chihuahua. En este caso se considera que el nivel de conocimientos políticos no se relaciona con la escolaridad, sino con el interés por estar informado. Bajo esa línea argumentativa, es posible señalar un desinterés juvenil por profundizar en temas de política: "En el aspecto escolar, se observa también un factor instructor en quienes tienen mayores años de escolaridad, ya que, para aquellos en nivel superior, 8 de cada 10 manifiestan desinterés en la política" (IMJUVE, 2013, p. 18).

Lo anterior permite explicar el uso de palabras específicas según escolaridad y ocupación para analizar la expresión de expectativas. La existencia de sofisticación no necesariamente implica la portación de un mayor nivel 
de conocimientos, sino el empleo de una jerga específica en el interior de cada grupo. No implica el conocimiento formal de las definiciones ni mucho menos la elección de la expresión adecuada conforme a los cánones normativos. La semejanza de los resultados de los árboles de similitud, tanto por nivel educativo como por ocupación, apuntan a la existencia de un vocabulario particular identificable por la pertenencia a una red caracterizada por los miembros que comparten la actividad, ya sea estudiar un nivel escolar o bien dedicarse a labores del hogar, la cual permite que se produzcan y reproduzcan expectativas en torno al voto. Aquello que Archer (2007) y Chalari (2009) llaman a tener presente, que es el proceso de coexistencia interactiva de los sujetos con otros, en el cual se trazan fronteras entre acciones y expectativas pertinentes y ajenas al grupo caracterizado por la ocupación o la escolaridad, por ejemplo. Dicho de otra forma, se argumenta que la conformación de las expectativas políticas de los jóvenes encuestados es interactiva dentro de un grupo de referencia (la universidad, la familia o el trabajo), compartiendo formas específicas de expresión relacionadas con lo que esperan de los políticos y los partidos políticos, en las cuales se deja huella de una mediación conflictiva (en referencia a la denominación de Chalari [2009]): los jóvenes no están conformes con la oferta electoral, por tanto sus expectativas subrayan la consecución de un cambio y no el rechazo tajante a los partidos políticos, ello bajo un contexto adverso en condiciones de inclusión desfavorables (Saraví, 2015).

El abstencionismo electoral juvenil es parte del comportamiento de las juventudes, que es multifactorial. En este caso se indagó la arista de las expectativas políticas de los abstencionistas juveniles, sin embargo, se reconoce que aún quedan cuestiones pendientes, sobre todo estudios longitudinales o modelajes para esclarecer las variables que explican la abstención de los jóvenes y con ello develar si estas se acompañan de formas particulares de asumir el abstencionismo en contextos específicos. 


\section{Referencias bibliográficas}

Almond, G. y S. Verba (1963). La cultura cívica. Princeton: Princeton University Press.

Anduiza, E. y A. Bosch (2004). Comportamiento político y electoral. Barcelona: Editorial Ariel.

Archer, M. (2007). Making our way through the world. Londres: Cambridge University Press.

Barry, B. (1970). Los sociólogos, los economistas y la democracia. Buenos Aires: Amorrortu.

Becerra, R. (1996). Participación política y ciudadana. En: J. A. Pérez (coord.). Jóvenes: una evaluación del conocimiento. Investigación sobre juventud en México. México: Causa Joven, pp. 529-603.

Bobbio, N. (2001). Diccionario de política. Vol. 1. México: Siglo XXI.

Boix, C. y C. Riba (2000). Las bases sociales del abstencionismo en las elecciones generales de España. Revista Española de Investigaciones Sociológicas, 90, pp. 95-128.

Burden, B. (2009). The dynamic effects of education on voter turnout. Electoral Studies, 28(4), pp. 540-549.

Camas, F. (2016). Así “votará" la abstención el 26-J. El País [en línea] Disponible en: $<$ http://politica.elpais.com/politica/2016/05/22/actualidad/1463931602_889741. $\mathrm{html}>$ [acceso 12/4/2016].

CESOP (2012). Jóvenes mexicanos en procesos electorales. En contexto, 21. Disponible en: <http://www3.diputados.gob.mx/camara/content/download/278305/871276/file/ Contexto-No.21-Jovenes-mexicanos-procesos-electorales.pdf $>$ [acceso 12/4/2016].

Chalari, A. (2009). Approaches to the individual: The relationship between Internal and External Conversation. Londres: Palgrave MacMillan.

Chalari, A. (2012). The causal powers of Social Change: the case of modern Greek society. Londres: Hellenic Observatory paper on Greece and Southeast Europe.

Chavarría, E. (2014). Relación entre desarrollo humano y abstencionismo a nivel local en Costa Rica para las elecciones del 2010. Revista de derecho electoral, 18, pp. 471-482.

CONAPO (2010). Mapas de marginación urbana de las zonas metropolitanas y ciudades de 100 mil o más habitantes 2010 [en línea]. Disponible en: <http://www.conapo. gob.mx/work/models/CONAPO/indices_margina/marginacion_urbana/AnexoA/ Documento/04A_AGEB.pdf $>$ [acceso 12/4/2017].

De Alba, M. (2004). El método Alceste y su aplicación al estudio de las representaciones sociales del espacio urbano: el caso de la Ciudad de México. Papers on Social Representations. Textes sur les représentations sociales, 13, pp. 1-20.

De la Paz, G. y R. Gómez (2012). Participación, instituciones y confianza: los jóvenes mexicanos frente al Instituto Federal Electoral, IFE [en línea]. Disponible en: $<$ https://portalanterior.ine.mx/archivos3/portal/historico/recursos/IFE-v2/CDD/ CDD-estructura/DOCS/Part_inst_20y_conf_jovenesIFE.pdf $>$ [acceso 12/4/2018].

De la Rosa, S. (2010). El abstencionismo en la elección del Ayuntamiento de Xalapa, en el periodo 1991-2000. [Tesis de licenciatura]. Xalapa: Universidad Veracruzana.

Downs, A. (1957). An economy theory of democracy. Nueva York: Harper Collins. 
Fernández, A. (2009). España-México: democracia, interés político y asociacionismo juvenil. El Cotidiano, 155, pp. 115-120.

Fernández, Y.; A. Mendieta y J. Castillo (2011). Análisis del abstencionismo y voto nulo en el proceso electoral de San Pedro Cholula 2010 [en línea]. Disponible en: <http:// www.ieepco.org.mx/biblioteca_digital/SOMEE\%202011/Área\%20temática\%204/ Mendieta,\%20Fernández\%20y\%20Castillo.\%20Análisis.pdf> [acceso 12/4/2017].

Fiorina, M. (1981). Retrospective Voting in American National Elections. New Haven: Yale University Press.

Garcés, A. (2010). De organizaciones a colectivos juveniles. Panorama de la participación política juvenil. Última década, 32, pp. 61-83.

Giraldo, C. A. (2017). Aproximación teórica al fenómeno del abstencionismo electoral. Conflicto \& Sociedad, 1(1), pp. 27-50.

Graham, C. (2010). Happiness Measures as a Guide to Development Policy? Promise and Potential Pitfalls [en línea]. Disponible en: <http://siteresources.worldbank. org/DEC/Resources/84797-1251813753820/6415739-1251815804823/Carol_Graham_paper.pdf $>$ [acceso 12/2/2018].

Hevia, F. (2012). Mas allá de las organizaciones civiles. Algunos problemas para caracterizar el fenómeno asociativo mexicano. En: V. Espinoza y A. Monsiváis (coords.). El deterioro de la democracia. Consideraciones sobre el régimen político, lo público y la ciudadanía en México. México: COLEF, pp. 367-394.

IDEA Internacional (2017). El estado de la democracia en el mundo 2017. Examen de la resiliencia democrática. Resumen. Estocolmo: IDEA Internacional [en línea]. Disponible en: <https://www.idea.int/gsod/files/IDEA-GSOD-2017-OVERVIEW-ES. pdf $>$ [acceso 12/2/2018].

IMJUVE (2013). Diagnóstico de la situación de los jóvenes mexicanos. México: IMJUVE-SEDESOL. Disponible en: <http://www.2012-2015.sedesol.gob.mx/work/models/SEDESOL/Resource/2936/1/images/Diagnostico_sobre_jovenes_en_Mexico. pdf $>$ [acceso 12/2/2018].

INE (2016). Estudio censal sobre la participación ciudadana en las elecciones del 2015. México: INE. Disponible en: <http://biblioteca.diputados.gob.mx/janium/bv/ ine/2016/estcen_parciu_elfed15.pdf $>$ [acceso 12/2/2018].

Jimenez, O. (2015). Los jóvenes y la democracia: retos y perspectivas para el ejercicio de su ciudadanía. Tla-melaua, 8(37), pp. 30-49.

Jiménez, Ó. (2009). La desilusión sexenal [en línea]. Disponible en $<$ http://www.eumed. net/tesis-doctorales/2012/oajm/ficha.htm> [acceso: 27/6/2017].

Latinobarómetro (2016). Informe 2016 [en línea]. Disponible en: <http://www.latinobarometro.org/latNewsShowMore.jsp?evYEAR=2016\&evMONTH=-1> [acceso $10 / 2 / 2018]$.

Latinobarómetro (2017). Informe 2017 [en línea]. Disponible en: <www.latinobarometro. org/LATDocs/F00006433-InfLatinobarometro2017.pdf> [acceso: 10/2/2018].

Lazarfeld, P.; B. Berelson y H. Gaudet (1962). El pueblo elige: estudio del proceso de formación del voto durante una campaña presidencial. Buenos Aires: Ediciones 3.

Leppäniemi, M.; H. Karjaluoto; H. Lehto y A. Goman (2010). Targeting Young Voters in a Political Campaign: Empirical Insights into an Interactive Digital Marketing Campaign. Journal of Nonprofit \& Public Sector Marketing, 22(1), pp. 14-37. 
López, P. (2016). Si se hubiera movilizado el voto de los "millenials", Hillary sería presidenta de los Estados Unidos. Trendencias [en línea]. Disponible en: <https://www. trendencias.com/redes-sociales/si-se-hubiera-movilizado-el-voto-de-los-millenialshillary-seria-presidenta-de-los-estados-unidos> [acceso: 12/5/2018].

Makowski, S. (2008). En la frontera de lo social: jóvenes y exclusión social. En: R. Cordera, P. Ramírez y A. Ziccardi (coords.). Pobreza, desigualdad y exclusión social en la ciudad del siglo XXI. México: Siglo XXI Editores-UNAM/IIS, pp. 168-181.

Maldonado, C. (2015). Participación política, apego a la democracia y temas prioritarios de las personas jóvenes en América Latina, 2000-2013. En: D. Trucco y H. Ullmann (eds.). Juventud: realidades y retos para un desarrollo con igualdad. Chile: CEPAL, pp. 207-259.

Mannarini, T.; M. Legittimo y C. Taló (2008). Determinants of social and political participation among youth. A preliminary study. Psicología Política, 36, pp. 95-117.

Marchand, P. y P. Ratinaud (2011). L'analyse de similitude appliquée aux corpus textuels: les primaires socialistes pour l'élection présidentielle française [en línea]. Disponible en: <http://lexicometrica.univ paris3.fr/jadt/jadt2012/Communications/Marchand, \%20Pascal\%20et $\% 20$ al. $\% 20-\% 20$ L $\% 27$ analyse $\% 20 \mathrm{de} \% 20$ similitude $\% 20$ appliquee \%20aux\%\%20textuels.pdf> [acceso: 12/2/2018].

Martínez, J. (2013). El abstencionismo electoral practicado por los ciudadanos jóvenes universitarios de entre 18 y 23 años de la ciudad de Orizaba, Veracruz, México, en los comicios para presidente municipal, 2000 y 2007. [Tesis de licenciatura]. Orizaba: Universidad Veracruzana.

Mizrahi, D. (2015). Los 10 países del mundo en los que menos gente va a votar. Infobae [en línea]. Disponible en: <http://www.infobae.com/2015/05/15/1728987-los10-paises-del-mundo-los-que-menos-gente-va-votar/> [acceso: 8/2/2018].

Molina, J. (2017). Tutorial para el análisis de textos con el software Iramuteq [en línea]. Disponible en: <https://www.researchgate.net/publication/315696508> [acceso: 18/2/2018].

Molina, J.; E. Barriga y V. Gámez (2017). Representaciones Sociales de los Adolescentes Catalanes sobre la Participación Política en Democracia. Revista Internacional de Sociología de la Educación, 6(1), pp. 85-109.

Morales, M.; H. Millán; M. Ávila y L. Fernández (2011). Participación y abstencionismo electoral en México. México: IFE, UAQ.

Murillo, C. y R. Pulido (2009). El abstencionismo en el estado de Chihuahua [en línea]. Disponible en: <https://es.slideshare.net/carmugo/el-abstencionismo-en-el-estadode-chihuahua $>$ [acceso: 12/2/2018].

Nohlen, D. (1994). Sistemas electorales y partidos políticos. México: FCE, UNAM.

Nohlen, D. (2003). Abstencionismo y participación electoral. Cuadernos de CAPEL, 49 [en línea]. Disponible en: <http://www2.congreso.gob.pe/sicr/cendocbib/con2 uibd.nsf/8118D723B4B646970525774300623D6C/\%24FILE/abstencionismo_participacion_electoral.pdf> [acceso: 12/2/2018].

North, D. (1989). Estructura y cambio en la historia económica. México: Fondo de Cultura Económica.

Ochoa, R. (2015). Comportamiento Electoral de los colombianos durante las elecciones presidenciales del año 2010. Jurídicas CUC, 11(1), pp. 209-220. 
OEA, Université Laval (2014). Estudio sobre participación electoral en América Central. Washington: SG/OEA.

OIJ (2013). El futuro ya llegó. $1^{\text {a }}$ Encuesta Iberoamericana de Juventudes. Informe Ejecutivo [en línea]. Disponible en: <http:/www.undp.org/content/undp/es/home/librarypage/democratic-governance/Encuesta_Iberoamericana_de_Juventudes.html $>$ [acceso: 5/2/2018].

Olesker, D. (2016). El mercado laboral en América Latina: pasado, presente y futuro. Nueva Sociedad [en línea]. Disponible en: <http://nuso.org/articulo/el-mercado-laboralen-america-latina-pasado-presente-y-futuro/> [acceso: 12/2/2018].

Oñate, T. (2010). El abstencionismo en México. Una visión institucional del tema. Alegatos, 74, pp. 257-266.

Padilla, H. (2010). Para mí es lo mismo. Causas del abstencionismo en Ciudad Juárez. Ciudad Juárez: Universidad Autónoma de Ciudad Juárez, Instituto Estatal Electoral Chihuahua.

Parales, C. y M. Vízcaíno (2007). Las relaciones entre actitudes y representaciones sociales: elementos para una integración conceptual. Revista Latinoamericana de Psicología, 39(2), pp. 351-361.

Reinert, M. (1986). Un logiciel d'analyse lexicale: Alceste. Cahiers de l'analyse des données, 11(4), pp. 471-481.

Rial, J. (2015). Post Scriptum sobre calidad de la democracia. En: L. Morlino. La calidad de las democracias en América Latina. Informe para IDEA Internacional. Costa Rica: IDEA, pp. 88-109.

Sander, P.; K. Stevenson; M. King y D. Coates (2000). University Students' Expectations of Teaching. Studies in Higher Education, 25(3), pp. 309-323.

Saraví, G. (2015). Juventudes fragmentadas. Socialización, clase y cultura en la construcción de la desigualdad. México: CIESAS/FLACSO.

Seagull, L. M. (1971). The Youth Vote and Change in American Politics. The Annals of the American Academy of Political and Social Science, 39(2), pp. 341-345.

Sota, I. (2016). Lo que pasa cuando no vas a votar. La Vanguardia [en línea]. Disponible en: <https://www.lavanguardia.com/vangdata/20160624/402737852941/brexitcuando-no-vas-a-votar-jovenes.html> [acceso: 12/4/2018].

Sulmont, D. (2010). Los enfoques teóricos sobre el comportamiento electoral [en línea]. $<$ http://escuela.pucp.edu.pe/gobierno/images/documentos/investigaciones/articu102_david_sulmont_22.pdf $>$ [acceso: 8/2/2018].

Wattenberg, M. P. (2003). Electoral turnout: The new generation gap. British Elections \& Parties Review, 13(1), pp. 159-173. 